\title{
Optimal Population Policy with Health Care and Lethal Pollution
}

\author{
Ulla Lehmijoki ${ }^{1} \cdot$ Tapio Palokangas $^{2}$ (D)
}

Received: 30 January 2020 / Accepted: 21 August 2021 / Published online: 1 October 2021

(C) The Author(s) 2021

\begin{abstract}
Optimal population policy is examined in the following setup. Families invest in capital, spend on health care and determine their number of children. Firms produce output from labor, capital and pollutants. Pollution increases, but private and public health care decrease mortality dynamically, with lags. Our main findings are the following. A marginal increase in public health care improves welfare as long as it diminishes the mortality rate more than that in private health care. The government can decentralize the social optimum by a parental tax on newborns and a Pigouvian tax on pollutants. Private health care should not be taxed.
\end{abstract}

Keywords Optimal taxation $\cdot$ Health care $\cdot$ Lethal pollution $\cdot$ Endogenous fertility and mortality

JEL J13 $\cdot \mathrm{O} 44 \cdot \mathrm{Q} 53 \cdot \mathrm{Q} 56 \cdot \mathrm{Q} 58$

\section{Introduction}

Although there is a lot of empirical evidence on the fact that pollution and health expenditures influence mortality, ${ }^{1}$ the studies on evironmental policy examine externalities related to fertility ${ }^{2}$, mortality ${ }^{3}$ or private health expenditures ${ }^{4}$ separately.

\footnotetext{
${ }^{1}$ Cf., Samet et al. (2000), Brunekreef and Holgate (2002), Pope et al. (2002), and Lehmijoki and Rovenskaya (2010).

${ }^{2}$ Cf., Harford (1997, 1998), Jöst and Quaas (2010), Baudin (2011) and De la Croix and Gosseries (2012).

3 Cf., Grossman (1972), Ehrlich and Chuma (1990) and Dalgaard and Strulik (2014).

4 Cf., Gomez (2001) and Baudin (2011).
}

Tapio Palokangas

tapio.palokangas@helsinki.fi

Ulla Lehmijoki

ulla.lehmijoki@helsinki.fi

1 University of Helsinki, IZA, Helsinki, Finland

2 University of Helsinki, HeGSE, IIASA, IZA, Helsinki, Finland 
The purpose of this document is to consider all those externalities in the same setup together with public health care. To do so, we apply optimal tax theory to a dynamic fertility choice model where production-related pollution is lethal, but where the families and the government can decrease mortality by spending on health care.

Harford (1997, 1998) examines optimal taxation in a model where altruistic parents determine the number of their children and population growth deteriorates the environment, decreasing an individual's welfare. He shows that to decentralize the social optimum, the Pigouvian tax on pollutants must be supported by a parental tax on the number of children. Jöst and Quaas (2010) examine optimal taxation in a fertility choice model where emissions generate welfare-harming pollution. They prove that the externalities must be corrected by two Pigouvian taxes: one on emissions and the other on the number of children or the size of the family. Harford (1997, 1998), assume a constant lifetime for an individual, while Jöst and Quaas (2010) assume a constant mortality rate (and consequently, a constant expected lifetime) for the whole population. In this document, the mortality rate is considered as an endogenous variable that depends on pollution along with private and public health care.

Jöst and Quaas (2010) assume furthermore that emissions are directly used as an input in production without costs. Consequently, there is no price for that input and no laissez-faire equilibrium in the economy without a tax on emissions. In this document, that problem is solved by considering emissions as a by-product of productive inputs that are extracted from the nature at a real cost.

Gomez (2001) and Baudin (2011) examine taxation in a fertility choice model where the families can reduce mortality by health expenditures. Both of them show that if private health expenditures generate positive externality, then it can be optimal to tax them. ${ }^{5}$ Because the public sector plays a major role in health care in most advanced countries, in this document, public services are considered as an alternative way of controlling that externality: private health care is a mortality-decreasing private good with no externality; but public health care can be either a public good whose average supply per individual decreases the mortality rate of the whole population, or a publicly-provided private good. This changes the whole setup: the problem is to discover the optimal supply of public health care and the supporting set of tax instruments by which the social optimum can be decentralized.

The evolution of the mortality rate is considered in many documents, but through the dynamics of some other state variable. Grossman (1972), and Erlich and Chuma (1990) introduce health as a mortality-decreasing state variable. Chakraborty (2004) assumes that the probability of an individual's survival depends on health capital that is augmented by public investment. Dalgaard and Strulik (2014) consider human aging as a process where the deficits of health are a state variable that increases the probability of death. In this document, pollution and health care are assumed to influence the mortality rate directly, through a dynamic process.

The remainder of this study is organized as follows. Section 2 presents the structure of the economy, including the behavior of the firms. Sections 3 derives

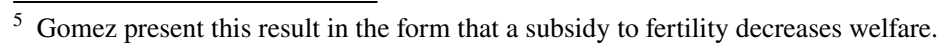


the social optimum by a model of the social planner (called the government), while Section 4 considers the optimal behavior of the representative family that determines its saving, fertility and private health care, taking the taxes, public health care and the stock of pollution as given. The comparison of the equilibria of the government and the family yields optimal policy rules for a market economy. Section 5 summarizes the results.

\section{The economy as a whole}

\subsection{The goods market}

In the model, time $t$ is continuous and $T$ is the initial time. There is one tradable good that is chosen as the numeraire in the model. It is assumed that pollutants $B$ - which are called carbon energy in this study, for convenience - are extracted from the nature at a constant marginal cost $q$ in terms of the numeraire good. Then, the market price for carbon energy is equal to $q$.

The output $Y$ of the good is used in consumption $C$, private health care $H$, public health care $G$, the extraction costs of carbon energy, $q B$, the depreciation of capital $K$ at the constant rate $\mu \in(0,1)$, and investment in new capital, $\frac{d K}{d t}$ :

$$
Y=C+H+G+q B+\mu K+\frac{d K}{d t}, \quad K(T)=K_{T},
$$

where $K_{T}$ is capital $K$ at initial time $T$.

It is convenient to define the macroeconomic variables and the labor input in production, $L$, in proportion to population $N$ as follows:

$$
c \doteq \frac{C}{N}, h \doteq \frac{H}{N}, k \doteq \frac{K}{N}, g \doteq \frac{G}{N}, y \doteq \frac{Y}{N}, b \doteq \frac{B}{N}, \ell \doteq \frac{L}{N} .
$$

Output $Y$ is produced from capital $K$, labor $L$ and carbon energy $B$ according to neoclassical technology with constant returns to scale. Then, by (2), the per individual output $y$ can be defined as a concave and linearly homogeneous function of per individual inputs $(k, \ell, b)$ :

$$
y(k, \ell, b), \quad y_{k} \doteq \frac{\partial y}{\partial k}>0, \quad y_{\ell} \doteq \frac{\partial y}{\partial \ell}>0, \quad y_{b} \doteq \frac{\partial y}{\partial b}>0 .
$$

\subsection{The government's budget}

Let $f$ be the fertility rate, i.e., the number of newborns relative to population $N$. The government controls the economy by public health care $G$ and the taxes $\tau_{f}$ 
and $\tau_{b}$ on fertility $f N$ and carbon energy $B$, and balances its budget by the poll tax $\tau_{n}$ on population $N$. This defines the budget constraint $\tau_{n} N+\tau_{f} f N+\tau_{b} q B=G$.

\subsection{Firms}

The representative firm maximizes its profit $\Pi$ by its inputs of capital $K$, labor $L$ and carbon energy $B$ subject to technology (3), given population $N$, the wage $w$, the interest rate $r$, and the price $q$ and the $\operatorname{tax} \tau_{b}$ on carbon energy $B$ [cf., (2) and (3)]:

$$
\Pi \doteq \max _{K, L, B}\left[Y-w L-r K-\left(q+\tau_{b}\right) B\right]=N \max _{k, \ell, b}\left[y(k, \ell, b)-w \ell-r k-\left(q+\tau_{b}\right) b\right] .
$$

Because the per individual production function (3) yields constant returns to scale, then, in equilibrium, the profit $\Pi$ is zero and the marginal products of capital, labor and carbon energy, $y_{k}, y_{\ell}$ and $y_{b}$, are equal to the interest rate $r$, the wage $w$ and the producer's unit cost of carbon energy, $q+\tau_{b}$, respectively:

$$
\Pi=0, \quad r=y_{k}, \quad w=y_{\ell}, \quad y_{b}=q+\tau_{b} .
$$

\subsection{Pollution and population growth}

The extraction and use of carbon energy $B$ generates emissions in one-to-one proportion. Emissions $B$ in turn accumulate the stock of pollution $P$ as follows [cf., (2)]:

$$
\frac{d P}{d t}=B-\xi P=b N-\xi P, \quad \xi>0, \quad P(T)=P_{T},
$$

where the constant $\xi$ is the natural absorbtion rate of pollution $P$.

At every moment of time $t$, each individual has a given stock of resources that can be allocated between labor $\ell$ and child rearing. Consequently, there is a transformation curve so that the individual has to sacrifice more and more labor $\ell$ to have one more child. Because pollution-induced illnesses decrease the individual's resources, pollution $P$ shifts that transformation curve downwards. Thus, the fertility rate $f$ is a decreasing function of both labor input $\ell$ and pollution $P$. The growth rate of population $N$ is then equal to the fertility rate $f$ minus the mortality rate $m$ as follows:

$$
\frac{1}{N} \frac{d N}{d t}=f(\ell, P)-m, \quad L(T)=L_{T}, \quad f_{\ell} \doteq \frac{\partial f}{\partial \ell}<0, \quad f_{P} \doteq \frac{\partial f}{\partial P}<0 .
$$

By (2) and (7), the change of capital per individual, $s \doteq \frac{d k}{d t}$, is determined as follows:

$$
s \doteq \frac{d k}{d t}=\frac{d}{d t}\left(\frac{K}{N}\right)=\frac{1}{N} \frac{d K}{d t}-\frac{K}{N^{2}} \frac{d N}{d t}=\frac{1}{N} \frac{d K}{d t}-(f-m) k, \quad k(T)=k_{T} .
$$




\subsection{Mortality}

Pollution $P$ is a negative public good that increases mortality for all individuals simultaneously. ${ }^{6}$ Private health care $H$ is a private good: its input to a single individual, $h$, decreases only that individual's mortality rate $m$. Public health care $G$ can be a public good as well, but then it is assumed to be proportional to population to maintain its efficiency. Hence, public health care per individual, $g$, decreases every individual's mortality rate $m$ simultaneously. Alternatively, public health care per individual, $g$, can be defined as a publicly-provided private good. Because the variables $(P, g, h)$ affect mortality over time with lags, the determination of the mortality rate $m$ can be specified as the following error-correcting process [cf., (2)]:

$$
\begin{aligned}
& \frac{d m}{d t}=\gamma[M(P, g, h)-m], \quad \gamma \in(0,1), \quad m(T)=m_{T}, \quad M_{P} \doteq \frac{\partial M}{\partial P}>0, \\
& M_{h} \doteq \frac{\partial M}{\partial h}<0, \quad M_{g} \doteq \frac{\partial M}{\partial g}<0, \quad M \text { strictly convex in }(g, h),
\end{aligned}
$$

where $M$ is the long-run mortality rate and $\gamma$ a constant adjustment parameter. $^{7}$

\subsection{Utility}

If an individual's probability of death depended explicitly on the individual's age, then the economy would become a non-autonomous system that depends explicitly on time. Because such a system does not have a steady state, it can be solved only numerically, but not symbolically by the standard methods of optimal control theory. To avoid this problem, we use the classical fertility choice model: ${ }^{8}$ the basic unit is not an individual, but a dynastic family - i.e., a sample of the whole population - that is able to keep its mortality and fertility rates stable over time.

In line with Razin and Ben-Zion (1975), Becker (1981), Galor and Weil (1996) and Stelter (2016), endogenous fertility is based on the following three assumptions. First, the parents are selfish. ${ }^{9}$ Second, children are a kind of consumption good in their parents' preferences. Finally, every individual's time horizon is limited by death, but, because the probability of dying is known, that individual discounts his/

\footnotetext{
6 According to many epidemiological studies (cf., Samet et al. 2000, Brunekreef and Holgate 2002, Pope et al. 2002, and Lehmijoki and Rovenskaya 2010), this holds true.

7 In the extreme special case with $\lim _{\gamma \rightarrow 1}$, the current mortality rate $m(t)$ would be a function of current pollution $P(t)$ and current health care $(h(t), g(t))$. Because the use of dynamic programming approximates the policy rules in the vicinity of the steady state, the results are the same also in that special case. The strict convexity of the long-term mortality rate $M$ in private and public health care per individual, $(h, g)$, ensures a unique equilibrium. It characterizes decreasing returns in controlling environmental damages.

${ }^{8}$ Cf., Becker (1981), Gomez (2001) and Jöst and Quaas (2010).

9 Because in the model capital per individual, $k=K / N$, is the only asset that a parent can leave to its offspring at the moment of his/her death, it is possible to introduce altruism into the model by placing $k$ as an argument into the utility function (10). This would slightly complicate the calculations, but, because the utility function $U$ would nevertheless be the same for both the government and the representative family, altruism brings no externality into the model and consequently, the results would be the same.
} 
her expected utility over time. In Appendix 1, it is shown that these three assumptions lead to the following utility function for the representative family in the economy: ${ }^{10}$

$$
\int_{T}^{\infty} U(c(t), f(\ell(t), P(t))) e^{[\rho+m(t)](T-t)} d t, \quad U_{c} \doteq \frac{\partial U}{\partial c}>0, \quad U_{f} \doteq \frac{\partial U}{\partial f}>0,
$$

where $c$ is consumption per individual, $f$ the fertility rate [cf., (7)], $\rho>0$ the constant rate of time preference for an individual who could live forever and $\rho+m$ is the effective rate of time preference for an individual that faces the mortality rate $m$.

\subsection{Transformation from real into virtual time}

Because the mortality rate $m$ is an endogenous variable, it must eliminated from the discount factor of the utility function (10) by Uzawa's (1968) transformation

$$
\theta(t)=[\rho+m(t)] t \text { with } d t=\frac{d \theta}{\rho+m(\theta)} .
$$

Because $\theta(T)=[\rho+m(T)] T, \theta(\infty)=\infty$ and $\frac{d t}{d \theta}=\frac{1}{\rho+m(\theta)}>0$ hold true, one can define $\theta(t)$ as an alternative virtual time variable and set the variables in terms of it. With the transformation (11), the utility function (10) and the evolution of the state variables, (6)-(9), are changed from real time $t$ into virtual time $\theta$ as follows:

$$
\begin{gathered}
\int_{\theta(T)}^{\infty} \frac{U(c(\theta), f(\ell(\theta), P(\theta)))}{\rho+m(\theta)} e^{\theta(T)-\theta} d \theta, \quad U_{c}>0, \quad U_{f}>0 \\
\frac{d N}{d \theta}=\frac{f(\ell(\theta), P(\theta))-m(\theta)}{\rho+m(\theta)} N(\theta), \quad N(T)=N_{T}, \quad f_{\ell}<0, \quad f_{P}<0 \\
\frac{d m}{d \theta}=\gamma \frac{M(P(\theta), g(\theta), h(\theta))-m}{\rho+m(\theta)}, \quad m(T)=m_{T}, \quad 0<\gamma<1, \quad M_{P}>0, \\
M_{h}<0, \quad M M_{g}<0, \quad \mathrm{M} \text { strictly convex in }(\mathrm{g}, \mathrm{h}), \\
\frac{d k}{d \theta}=\frac{s(\theta)}{\rho+m(\theta)}, \quad k(T)=k_{T}, \\
\frac{d P}{d \theta}=\frac{b(\theta) N(\theta)-\xi P(\theta)}{\rho+m(\theta)}, \quad P(T)=P_{T}, \quad 0<\xi<1 .
\end{gathered}
$$

\footnotetext{
${ }^{10}$ It is also possible to define an individual's temporary utility $U$ as a function of that individual's mortality rate $m$ as well. Because then the function $U$ would still be the same for the family and the government, the results would nevertheless be the same as in the current version.
} 


\section{The government}

\subsection{Optimal behavior}

To obtain the first-best optimum, it is assumed that the government can control the economy by the per individual amounts of the change of capital, $s \doteq \frac{d k}{d t}$, labor input $\ell$, public health care $g$, private health expenditures $h$ and carbon energy $b$. Noting (1), (2), (3), (5), (7) and (8), consumption per individual, $c$, can be derived as a function the government's controls $(s, \ell, g, h, b)$ and state variables $(m, k, P)$ as follows:

$$
\begin{aligned}
& s=\frac{1}{N} \frac{d K}{d t}-(f-m) k=y-c-h-g-q b+(m-f-\mu) k \Leftrightarrow \\
& c(s, \ell, g, h, b, m, k, P) \doteq y(k, \ell, b)-s-h-g-q b+[m-f(\ell, P)-\mu] k \text { with } \\
& \frac{\partial c}{\partial b}=y_{b}-q=\tau_{b}, \frac{\partial c}{\partial \ell}=y_{\ell}-k f_{\ell}=w-k f_{\ell}, \frac{\partial c}{\partial s}=\frac{\partial c}{\partial h}=\frac{\partial c}{\partial g}=-1, \\
& \frac{\partial c}{\partial m}=k \text { and } \frac{\partial c}{\partial k}=y_{k}+m-f-\mu=r+m-f-\mu .
\end{aligned}
$$

In Appendix 2, the marginal cost of pollution $P$ - when the long-run mortality rate $M$, population $N$, capital stock $K$, emissions $B$, public health care $G$ and labor input per individual, $\ell$, are held constant - is derived as follows:

$$
\varepsilon=N\left|\frac{M_{P}}{M_{h}}\right|-K\left|\frac{\partial f}{\partial P}\right|=-N \underbrace{\frac{M_{P}}{M_{h}}}_{-}+K \underbrace{\frac{\partial f}{\partial P}}_{-} .
$$

Because of two opposing effects, the sign of this marginal cost is ambiguous. On the one hand, an increase in pollution $P$ increases the long-run mortality rate by the amount $M_{P}$, compelling the families to increase their private health care by the amount $\left|N M_{P} / M_{h}\right|$. On the other hand, pollution $P$ decreases the fertility rate by the amount $\left|\frac{\partial f}{\partial P}\right|$, reducing investment in new machines for the newborns by the amount $K\left|\frac{\partial f}{\partial P}\right|$. It is assumed that the positive effect through the long-run mortality rate $M$ dominates over the negative effect through the fertility rate $f$, so that $\epsilon>0$. Otherwise, we would obtain the unlikely case that pollution is not a social loss. ${ }^{11}$

It is convenient to define the social cost of pollution, $\eta$, as the value of the flow of the marginal cost of pollution, $\varepsilon$, discounted by the sum of the natural absorbtion rate of pollution, $\xi$, and the effective rate of time preference, $\rho+m$ :

$$
\eta \doteq \frac{\varepsilon}{\xi+\rho+m}>0
$$

The faster the stock of pollution disappears (i.e., the higher $\xi$ ) or the higher an individual's effective rate of time preference, $\rho+\mathrm{m}$, is, the less worried the individuals are about pollution $P$ and the lower the social cost of pollution, $\eta$, is.

\footnotetext{
${ }^{11}$ If $\epsilon<0$ held true, then, by the definition (19) and Propositions 1 and 2, both fertility and the use of carbon energy should be subsidized, $\tau_{f}<0$ and $\tau_{b}<0$.
} 
The government maximizes the representative family's welfare (12) by its controls $(s, \ell, g, h, b)$ subject to consumption per individual, (17), and the evolution of population $N$, the mortality rate $m$, capital per individual, $k$, and pollution $P$, (13)-(16). The value function for the government's program at initial time $T$ is then

$$
\Psi(m, k, N, P, T) \doteq \max _{(s(T), \ell(T), h(T), g(T), b(T))} \operatorname{s.t.(17),(13)-(16)}_{\theta(T)}^{\infty} \frac{U(c(\theta), f(\ell(\theta), P(\theta)))}{\rho+m(\theta)} e^{T-\theta} d \theta .
$$

\subsection{Equilibrium conditions}

Following Dixit and Pindyck (1994), the Bellman equation for the government's program (20) is constructed as follows:

$$
\begin{gathered}
\Psi=\underset{(s(T), \ell(T), h(T), g(T), b(T)) s . t .(17)}{\max } \Upsilon(s, \ell, h, b, m, k, N, P, T) \text { with } \\
\Upsilon(s, \ell, h, g, b, m, k, N, P, T) \doteq \frac{U}{\rho+m}+\frac{\partial \Psi}{\partial N} \frac{d N}{d \theta}+\frac{\partial \Psi}{\partial P} \frac{d P}{d \theta}+\frac{\partial \Psi}{\partial k} \frac{d k}{d \theta}+\frac{\partial \Psi}{\partial m} \frac{d m}{d \theta} \\
=\frac{1}{\rho+m}\left\{U(c, f(\ell, P))+\frac{\partial \Psi}{\partial N}[f(\ell, P)-m] N+\frac{\partial \Psi}{\partial P}(b N-\xi P)\right. \\
\left.+\frac{\partial \Psi}{\partial k} s+\frac{\partial \Psi}{\partial m} \gamma[M(P, g, h)-m]\right\} .
\end{gathered}
$$

The maximization of the function (22) by controls subject to the consumption function (17) yields the first-order conditions (cf., Appendix 3)

$$
\begin{gathered}
M_{g}=\frac{U_{c}}{\gamma} / \frac{\partial \Psi}{\partial m}, \\
M_{h}=\frac{U_{c}}{\gamma} / \frac{\partial \Psi}{\partial m}, \\
U_{c}=\frac{\partial \Psi}{\partial k}, \\
\tau_{b}=\frac{\partial c}{\partial b}=-\frac{N}{U_{c}} \frac{\partial \Psi}{\partial P}, \\
\frac{U_{f}}{U_{c}}+\frac{w}{f_{\ell}}-k=-\frac{N}{U_{c}} \frac{\partial \Psi}{\partial N} .
\end{gathered}
$$


Because $\Upsilon$ is concave in controls $(s, \ell, h, g, b)$, the government's equilibrium is unique.

From (23) and (24) it follows that $M_{g}=M_{h}$. Then, the marginal effects of $H$ and $G$ on $M(P, g, h)$, when $P$ and $N$ are held constant, are given by $M_{h} \frac{\partial h}{\partial H}=\frac{M_{h}}{N}<0$ and

$$
M_{g} \frac{\partial g}{\partial H}=\frac{M_{g}}{N}=\frac{M_{h}}{N}=M_{h} \frac{\partial h}{\partial H}<0 .
$$

This result can be rephrased as follows:

Proposition 1 Public health care $G$ must be extended up to the level at which its marginal efficiency in decreasing the long-run mortality rate $M,-M_{g} \frac{\partial g}{\partial H}$, is equal to that of private health care $H,-M_{h} \frac{\partial g}{\partial H}$, given pollution $P$ and population $N$.

Because both private and public health care are produced from the final good to decrease the mortality rate, efficiency requires that their marginal effects are equal.

\subsection{Solution}

The solution of dynamic programming is based on finding a proper specification for the value function (20). In Appendix 4, the following function is found:

$$
\begin{aligned}
& \Psi(m, k, N, P, T), \quad \frac{\partial \Psi}{\partial P}=\frac{U_{c}}{\rho+m} \frac{\xi \tau_{b}-\epsilon}{N}, \quad \frac{\partial \Psi}{\partial N}=-\frac{\tau_{b} U_{c}}{\rho+m} \frac{b}{N}, \\
& \frac{\partial \Psi}{\partial m}=\frac{1}{\rho+m}\left[U_{f}+U_{c}\left(k-\frac{1}{M_{h}}\right)\right]-\frac{U}{(\rho+m)^{2}} .
\end{aligned}
$$

Noting (19) and (28), condition (26) becomes

$$
\tau_{b}=-\frac{N}{U_{c}} \frac{\partial \Psi}{\partial P}=\frac{\varepsilon-\xi \tau_{b}}{\rho+m} \Leftrightarrow(\xi+\rho+m) \tau_{b}=\varepsilon \Leftrightarrow \tau_{b}=\frac{\varepsilon}{\xi+\rho+m}=\eta>0 .
$$

This result can be rephrased as follows:

Proposition 2 The optimal tax on carbon energy is equal to the social cost of pollution, $\tau_{b}=\eta>0$.

\section{The representative family}

\subsection{Saving}

The representative family takes pollution $P$, the $\operatorname{tax} \tau_{n}$ on population $N$, the tax $\tau_{f}$ on fertility $f N$, public health care per individual, $g$, the wage $w$ for labor $L$ and the interest rate $r$ paid to capital $K$ as given. Because capital $K$ is the only asset in the model, investment $\frac{d K}{d t}$ in capital $K$ is equal to saving: 


$$
\frac{d K}{d t}=w L+r K-C-H-\left(\tau_{n} N+\tau_{f} f N\right)-\mu K,
$$

where $w L$ is labor income, $r K$ capital income, $C$ consumption, $H$ private health care, $\tau_{n} N+\tau_{f} f N$ the family's tax expenditures and $\mu$ the constant rate of depreciation for capital $K$.

The family determines the change of its capital per individual, $s=\frac{d k}{d t}$, labor input per individual, $\ell$, and health expenditures per individual $h$ subject to the evolution of its mortality rate $m,(14)$, and that of its capital per individual, $k,(15)$. By (2), (7), (8) and (29), consumption per individual, $c$, is a function of the family's controls $(s, \ell, h)$, state variables $(m, k)$, and exogenous variables $\left(P, g, \tau_{n}, \tau_{f}, w, r\right)$ (cf., Appendix 5):

$$
\begin{aligned}
& c=\widetilde{c}\left(s, \ell, h, m, k, P, g, \tau_{n}, \tau_{f}, w, r\right), \quad \frac{\partial \widetilde{c}}{\partial m}=-k, \quad \frac{\partial \widetilde{c}}{\partial s}=\frac{\partial \widetilde{c}}{\partial h}=-1, \\
& \frac{\partial \widetilde{c}}{\partial \ell}=w-\left(k+\tau_{f}\right) f_{\ell}, \quad \frac{\partial \widetilde{c}}{\partial k}=m-f+r-\mu .
\end{aligned}
$$

\subsection{Optimal behavior}

The representative family maximizes its utility (12) by its controls $(s, \ell, h)$ subject to its consumption per individual, (30), the evolution of its mortality rate, (14), and its capital per individual, (15), given its exogenous variables $\left(P, \tau_{n}, \tau_{f}, g, w, r\right)$. This defines the value function at initial time $T$ as follows:

$$
\Phi\left(m, k, P, \tau_{n}, \tau_{f}, w, r, g, T\right) \doteq \max _{(s(T), \ell(T), h(T)) \text { s.t. (30),(14),(15) }} \int_{\theta(T)}^{\infty} \frac{U(c(\theta), f(\ell(\theta), P(\theta)))}{\rho+m(\theta)} e^{\theta(T)-\theta} d \theta .
$$

The family's program (31) is independent of the family size $N$.

The Bellman equation for the family's program (31) is given by

$$
\begin{aligned}
& \Phi\left(m, k, P, \tau_{n}, \tau_{f}, w, r, g, T\right)=\max _{(s(T), \ell(T), h(T)) \text { s.t. }(30),(14),(15)} \Lambda\left(s, \ell, h, m, k, P, \tau_{n}, \tau_{f}, w, r, g, T\right) \text { with } \\
& \Lambda\left(s, \ell, h, m, k, P, \tau_{n}, \tau_{f}, w, r, g, T\right) \doteq \frac{U}{\rho+m}+\frac{\partial \Phi}{\partial k} \frac{d k}{d \theta}+\frac{\partial \Phi}{\partial m} \frac{d m}{d \theta} \\
& =\frac{1}{\rho+m}\left\{U+\frac{\partial \Phi}{\partial k} s+\gamma \frac{\partial \Phi}{\partial m}[M(P, g, h)-m]\right\} .
\end{aligned}
$$

The maximization of the function (32) by the controls $(s, \ell, h)$ subject to the family's consumption function (30) yields the first-oder conditions (cf., Appendix 6)

$$
\begin{gathered}
M_{h}=\frac{U_{c}}{\gamma} / \frac{\partial \Phi}{\partial m}, \\
U_{c}=\frac{\partial \Phi}{\partial k},
\end{gathered}
$$




$$
\tau_{f}=\frac{1}{f_{\ell}} \frac{U_{f}}{U_{c}}+\frac{w}{f_{\ell}}-k .
$$

Because $\Lambda$ is strictly concave in the controls $(s, f, h)$, that equilibrium is unique.

\subsection{Solution}

The solution of dynamic programming is based on finding a specification for the value function (31). In Appendix 7, the following function is found:

$$
\Phi\left(m, k, P, \tau_{n}, \tau_{f}, w, r, g, T\right), \quad \frac{\partial \Phi}{\partial k}=\frac{\partial \Psi}{\partial k}, \quad \frac{\partial \Phi}{\partial m}=\frac{\partial \Psi}{\partial m} .
$$

Inserting the partial derivatives in (36) into the family's first-order conditions (33) and (34) yields the government's first-order conditions (24) and (25).

The optimal parental tax $\tau_{f}$ can be derived from (28), Proposition 2, the government's first-order condition (27) and the family's first-order condition (35) as follows:

$$
\tau_{f}=\frac{1}{f_{\ell}} \frac{U_{f}}{U_{c}}+\frac{w}{f_{\ell}}-k=-\frac{N}{U_{c}} \frac{\partial \Psi}{\partial N}=\frac{\tau_{b} b}{\rho+m}=\frac{b \eta}{\rho+m}>0 .
$$

This result can be rephrased as follows:

Proposition 3 The optimal parental tax per newborn, $\tau_{f}$, is equal to the product of carbon energy per individual, $b$, and the social cost of pollution, $\eta$, discounted by an individual's effective rate of time preference, $\rho+m$.

The product of carbon energy per newborn (= carbon energy per individual) and the social cost of pollution, $b \eta$, characterizes the social cost of a newborn through pollution at a moment of time. The parental tax per newborn, $\tau_{f}$, must be equal to the value of the social cost of a newborn over time, $b \eta$, discounted an individual's effective rate of time preference, $\rho+m$.

\section{Concluding remarks}

In this document, optimal public policy is constructed for a market economy with the following institutions. Firms produce output from labor, capital and pollutants. Families determine their saving, fertility and private health expenditures. Emissions are a by-product of the extraction and use of pollutants. The mortality rate depends on pollution and both private and public health care dynamically, with lags. The government can use linear taxes and public health care to control the economy.

In the literature, the authors that combine endogenous fertility with environmental problems commonly recommend corrective Pigouvian taxes, but assuming 
in their models that the mortality rate (or longevity) is exogenous and there is no health care. ${ }^{12}$ Furthermore, Gomez (2001) and Bauldin (2011) suggest that if there is externality in health care, then it can be optimal to tax private health expenditures. In this document, the main results are the following. If public health care can be introduced to take care of externality in health care, then private health expenditures should not be taxed or subsidized. If public health care uses the same inputs as private health care, then its expansion is welfare improving up to the level at which its marginal efficiency in decreasing the mortality rate is equal to that of private health care, given pollution and population. With this health care policy, two taxes - one on newborns and the other on pollutants - are sufficient to decentralize the social optimum. These stabilize the population and eliminate the externality due to pollution.

To construct the optimality rules for the taxes on newborns and pollutants, information on two concepts is needed. The first of these is the marginal cost of pollution on the assumption that the long-run mortality rate, population, capital stock, emissions, public health care and labor input relative to population are held constant. The second is the social cost of pollution, i.e., the value of the flow of the marginal cost of pollution, discounted by the sum of the natural absorbtion rate of pollution, the mortality rate and the rate of time preference. The optimal tax on pollutants is equal to the social cost of pollution. The optimal parental tax per newborn is equal to the product of carbon energy per newborn and the social cost of pollution, discounted by the sum of the mortality rate and the rate of time preference. These result can be explained as follows. The greater the mortality rate, the rate of time preference or the natural absorbtion rate of pollution, the less worried the families are about future pollution. Consequently, the social optimum can be decentralized by smaller corrective taxes.

\section{Appendix}

\section{The utility function (Eq. 10)}

It is assumed that at any time, $t$, an individual derives its temporary utility $U$ from consumption per head, $c \doteq C / N$, and the fertility rate, $f$, in its family through the strictly concave function $U(c, f)$. Plugging the fertility function from (7) into that function yields

$$
U(c(t), f(\ell(t), P(t))), \quad U_{c} \doteq \frac{\partial U}{\partial c}>0, \quad U_{f} \doteq \frac{\partial U}{\partial f}>0 .
$$

Because the mortality rate is $m$, the probability of dying in a short time $d t$ is equal to $m d t$, the probability that an individual will survive beyond the period $[T, t]$, is given by $e^{(T-t) m(t)}$, and the individual's expected utility at time $t \mathrm{~s}$ given by

12 Cf., Harford (1997, 1998), Jöst and Quaas (2009) and De la Croix and Gosseries (2012). 


$$
e^{(T-t) m(t)} U(c(t), f(\ell(t), P(t))) .
$$

The utility function of the representative family is then the expected utility of the representative member of that family for the planning period $t \in[0, \infty)$ as follows: ${ }^{13}$

$$
\int_{T}^{\infty}\left(U e^{m(T-t)}\right) e^{\rho(T-t)} d t=\int_{T}^{\infty} U(c(t), f(\ell(t), P(t))) e^{[\rho+m(t)](T-t)} d t, \quad \rho>0,
$$

where $\rho$ is the constant rate of time preference for an individual who could live forever.

\section{The marginal cost of pollution (Eq. 18)}

Because any costs due to pollution $P$ deprive crowd out consumption $C$, the marginal cost of pollution can defined by $\varepsilon \doteq-\frac{\partial C}{\partial P}$. Keeping the long-run mortality rate $M$, population $N$, capital stock $K$, emissions $B$, public health care $G$ and labor input per individual, $\ell$, constant, from (2), (7), (9) and (17) it follows that

$$
\begin{aligned}
\varepsilon & \doteq-\left.\frac{\partial C}{\partial P}\right|_{(M, N, K, B, G, \ell) \text { constants }}=-\left.N \frac{\partial c}{\partial P}\right|_{(M, K, k, b, g, \ell) \text { constants }, s=0} \\
& =N\left(k \frac{\partial f}{\partial P}+\left.\frac{\partial h}{\partial P}\right|_{M(P, g, h)=\text { constant }}\right)=K \underbrace{\frac{\partial f}{\partial P}}_{-}-N \underbrace{\frac{M_{P}}{M_{h}}}_{-} .
\end{aligned}
$$

\section{The government's first-order conditions (Eqs 23-27)}

The maximization (21) by controls $(s, \ell, h, g, b)$ subject to (17) and (22) yields the first-order conditions

$$
\begin{aligned}
& 0=\frac{\partial \Upsilon}{\partial b}=\frac{1}{\rho+m}\left(U_{c} \frac{\partial c}{\partial b}+\frac{\partial \Psi}{\partial P} N\right)=\frac{1}{\rho+m}\left(U_{c} \tau_{b}+\frac{\partial \Psi}{\partial P} N\right) \Leftrightarrow \tau_{b}=-\frac{N}{U_{c}} \frac{\partial \Psi}{\partial P}, \\
& 0=\frac{\partial \Upsilon}{\partial s}=\frac{1}{\rho+m}\left(U_{c} \frac{\partial c}{\partial s}+\frac{\partial \Psi}{\partial k}\right)=\frac{1}{\rho+m}\left(-U_{c}+\frac{\partial \Psi}{\partial k}\right) \Leftrightarrow U_{c}=\frac{\partial \Psi}{\partial k}, \\
& 0=\frac{\partial \Upsilon}{\partial \ell}=\frac{1}{\rho+m}\left(U_{c} \frac{\partial c}{\partial \ell}+U_{f} f_{\ell}+\frac{\partial \Psi}{\partial N} N f_{\ell}\right)=\frac{U_{c} f_{\ell}}{\rho+m}\left(\frac{1}{f_{\ell}} \frac{\partial c}{\partial \ell}+\frac{U_{f}}{U_{c}}+\frac{\partial \Psi}{\partial N} \frac{N}{U_{c}}\right) \\
& =\frac{U_{c}}{\rho+m}\left(\frac{w}{f_{\ell}}-k+\frac{U_{f}}{U_{c}}+\frac{\partial \Psi}{\partial N} \frac{N}{U_{c}}\right) \Leftrightarrow \frac{U_{f}}{U_{c}}+\frac{w}{f_{\ell}}-k=-\frac{N}{U_{c}} \frac{\partial \Psi}{\partial N}, \\
& 0=\frac{\partial \Upsilon}{\partial h}=\frac{1}{\rho+m}\left(U_{c} \frac{\partial c}{\partial h}+\frac{\partial \Psi}{\partial m} \gamma M_{h}\right)=\frac{1}{\rho+m}\left(\frac{\partial \Psi}{\partial m} \gamma M_{h}-U_{c}\right) \Leftrightarrow M_{h}=\frac{U_{c}}{\gamma} / \frac{\partial \Psi}{\partial m}, \\
& 0=\frac{\partial \Upsilon}{\partial g}=\frac{1}{\rho+m}\left(U_{c} \frac{\partial c}{\partial g}+\frac{\partial \Psi}{\partial m} \gamma M_{g}\right)=\frac{1}{\rho+m}\left(\frac{\partial \Psi}{\partial m} \gamma M_{g}-U_{c}\right) \Leftrightarrow M_{g}=\frac{U_{c}}{\gamma} / \frac{\partial \Psi}{\partial m} .
\end{aligned}
$$

\footnotetext{
13 According to Jöst and Quaas (2010), the alternative way of defining the utility function of the representative family could be the sum of the utilities of all members of that family, $\int_{0}^{\infty}\left(N U e^{-m t}\right) e^{-\rho t} d t$. That definition, however, makes social welfare artificially dependent of the size of the family, $N$, while the arithmetic average of the members' utilities leads back to the definition (37).
} 


\section{The government's value function (Eq. 28)}

In the steady state of the system (13)-(16), it holds true that

$$
\frac{d k}{d \theta}=s=0, \quad \frac{d N}{d \theta}=0 \Leftrightarrow f(\ell, P)=m, \quad \frac{d P}{d \theta}=0 \Leftrightarrow b=\xi \frac{P}{N}, \quad \frac{d m}{d \theta}=0 \Leftrightarrow M(P, g, h)=m .
$$

The condition $M(P, g, \bar{h})=m$ defines the steady-state private health care per individual, $\bar{h}$, as a function of the mortality rate $m$, public health care $g$ and pollution $P$ as follows [cf., (13) and (14)]:

$$
\bar{h}(m, g, P), \quad \frac{\partial \bar{h}}{\partial m}=\frac{1}{M_{h}}, \quad \frac{\partial \bar{h}}{\partial g}=-\frac{M_{g}}{M_{h}}<0, \quad \frac{\partial \bar{h}}{\partial P}=-\frac{M_{P}}{M_{h}}>0 .
$$

By (2), (5), (17), (18), (38) and (39), steady-state consumption per individual, $\bar{c}$, is defined as the following function of the mortality rate $m$, capital per individual, $k$, and pollution $P$ :

$$
\begin{aligned}
& \bar{c}(m, k, P, N) \doteq \max _{\ell, g}\{y(k, \ell, b)-s-\bar{h}(m, g, P)-g-q b+[m-f(\ell, P)-\mu] k\}_{s=0, b=\xi \frac{P}{N}} \\
& =\left\{\max _{\ell}[y(k, \ell, b)-f(\ell, P) k]-\min _{g}[\bar{h}(m, g, P)+g]-q b+(m-\mu) k\right\}_{s=0, b=\xi \frac{P}{N}} \text { with } \\
& \frac{\partial \bar{c}}{\partial m}=k-\frac{\partial \bar{h}}{\partial m}=k-\frac{1}{M_{h}},\left.\quad \frac{\partial \bar{c}}{\partial k}\right|_{f=m}=\left(y_{k}-f+m-\mu\right)_{f=m}=r-\mu, \\
& \frac{\partial \bar{c}}{\partial P}=\left.\left(y_{b}-q\right) \frac{\partial b}{\partial P}\right|_{b=\xi \frac{P}{N}}-\frac{\partial f}{\partial P} k-\frac{\partial \bar{h}}{\partial P}=\tau_{b} \frac{\xi}{N}-\frac{\partial f}{\partial P} k-\frac{\partial \bar{h}}{\partial P}=\frac{1}{N}\left(\xi \tau_{b}-\frac{\partial f}{\partial P} K-\frac{\partial \bar{h}}{\partial P} N\right)=\frac{\xi \tau_{b}-\epsilon}{N}, \\
& \text { and } \quad \frac{\partial \bar{c}}{\partial N}=\left.\left(y_{b}-q\right) \frac{\partial b}{\partial N}\right|_{b=\xi \frac{P}{N}}=\left.\tau_{b} \frac{\partial b}{\partial N}\right|_{b=\xi \frac{P}{N}}=-\left.\tau_{b} \frac{\xi P}{N^{2}}\right|_{b=\xi \frac{P}{N}}=-\tau_{b} \frac{b}{N} .
\end{aligned}
$$

The solution of dynamic programming is based on finding a specification for the value function $\Psi$ that is defined by (28). Because the optimal policy must lead the system to the steady state (38), the value function $\Psi$ must hold true in the steady state. Noting (21) and (22), this hint leads to

$$
\left.\left.\Psi\right|_{(38)} \doteq \max _{s, \ell, h, g, b} \Upsilon\right|_{(38)}=\left.\max _{g} \Upsilon\right|_{s=0, f=m, \ell=\bar{\ell}, h=\bar{h}, b=\xi \frac{P}{N}}=\frac{U(\bar{c}, m, P, k)}{\rho+m}
$$

Thus, one can examine whether the value function

$$
\Psi(m, k, N, P, T)=\left.\max _{s, \ell, h, g, b} \frac{U(c, f, P, k)}{\rho+m}\right|_{(38)}=\frac{U(\bar{c}, m, P, k)}{\rho+m}
$$

solves the problem. Noting (40) and (18), the partial derivatives of the function (41) with respect to the state variables $(N, P, m, k)$ are given by 


$$
\begin{aligned}
& \frac{\partial \Psi}{\partial k}=\frac{U_{c}}{\rho+m} \frac{\partial \bar{c}}{\partial k}=\frac{U_{c}(r-\mu)}{\rho+m}, \frac{\partial \Psi}{\partial P}=\frac{U_{c}}{\rho+m} \frac{\partial \bar{c}}{\partial P}=\frac{U_{c}}{\rho+m} \frac{\xi \tau_{b}-\epsilon}{N}, \frac{\partial \Psi}{\partial N}=\frac{U_{c}}{\rho+m} \frac{\partial \bar{c}}{\partial N}=-\frac{\tau_{b} U_{c}}{\rho+m} \frac{b}{N} \\
& \frac{\partial \Psi}{\partial m}=\frac{1}{\rho+m}\left(U_{c} \frac{\partial \bar{c}}{\partial m}+U_{f}\right)-\frac{U}{(\rho+m)^{2}}=\frac{1}{\rho+m}\left[U_{c}\left(k-\frac{1}{M_{h}}\right)+U_{f}\right]-\frac{U}{(\rho+m)^{2}}
\end{aligned}
$$

\section{The family's consumption function (Eq. 30)}

By Eqs. (2), (7), (8) and (29) the function (30) can be defined as follows:

$$
\begin{aligned}
& s=\frac{1}{N} \frac{d K}{d t}-(f-m) k=\left[w L+r K-C-H-\tau_{n} N-\tau_{f} f N-\mu K\right] / N-(f-m) k \\
& =w \ell+r k-c-h-\tau_{n}-\tau_{f} f(\ell, P)-\mu k-[f(\ell, P)-m] k \Leftrightarrow \\
& c=\widetilde{c}\left(s, \ell, h, m, k, P, g, \tau_{n}, \tau_{f}, w, r\right) \doteq w \ell+r k-s-h-\tau_{n}-\tau_{f} f(\ell, P)-\mu k-[f(\ell, P)-m] k .
\end{aligned}
$$

This function has the partial derivatives

$$
\frac{\partial \widetilde{c}}{\partial m}=-k, \quad \frac{\partial \widetilde{c}}{\partial s}=\frac{\partial \widetilde{c}}{\partial h}=-1, \frac{\partial \widetilde{c}}{\partial \ell}=w-\left(k+\tau_{f}\right) f_{\ell}, \frac{\partial \widetilde{c}}{\partial k}=m-f+r-\mu .
$$

\section{The family's first-order conditions (Eqs. 23-27)}

The first-order conditions for maximizing the function Eq. (32) by the controls $(s, f, h)$ subject to Eq. (30) are

$$
\begin{aligned}
& 0=\frac{\partial \Lambda}{\partial s}=\frac{1}{\rho+m}\left(U_{c} \frac{\partial \widetilde{c}}{\partial s}+\frac{\partial \Phi}{\partial k}\right)=\frac{1}{\rho+m}\left(\frac{\partial \Phi}{\partial k}-U_{c}\right) \Leftrightarrow U_{c}=\frac{\partial \Phi}{\partial k} \\
& 0=\frac{\partial \Lambda}{\partial \ell}=\frac{1}{\rho+m}\left(U_{c} \frac{\partial \widetilde{c}}{\partial \ell}+U_{f}\right)=\frac{U_{c}}{\rho+m}\left[w-\left(k+\tau_{f}\right) f_{\ell}+\frac{U_{f}}{U_{c}}\right] \Leftrightarrow \tau_{f}=\frac{1}{f_{\ell}} \frac{U_{f}}{U_{c}}-k+\frac{w}{f_{\ell}}, \\
& 0=\frac{\partial \Lambda}{\partial h}=\frac{1}{\rho+m}\left(U_{c} \frac{\partial \widetilde{c}}{\partial h}+\gamma M_{h} \frac{\partial \Phi}{\partial m}\right)=\frac{1}{\rho+m}\left(-U_{c}+\gamma M_{h} \frac{\partial \Phi}{\partial m}\right) \Leftrightarrow M_{h}=\frac{U_{c}}{\gamma} / \frac{\partial \Phi}{\partial m}
\end{aligned}
$$

\section{The family's value function (Eq. 36)}

In the steady state of the system Eqs. (14) and (15), it holds true that

$$
\frac{d k}{d \theta}=s=0, \quad \frac{d m}{d \theta}=0 \Leftrightarrow M(P, g, h)=m
$$

The last of these conditions, $M(P, g, h)=m$, defines the function

$$
\widetilde{h}(m, g, P), \quad \frac{\partial \tilde{h}}{\partial m}=\frac{1}{M_{h}} .
$$


By Eqs. (30) and (43) steady-state consumption per individual, $\widehat{c}$, can be defined as a function of the fertility rate $f$, the mortality rate $m$, capital per individual, $k$, public health care per individual, $g$, and pollution $P$ :

$$
\begin{aligned}
& \widehat{c}=\left.\widetilde{c}\right|_{(42)}=w(1-f)+r k-\widetilde{h}(m, g, P)-\tau_{n}+(m-f-\mu) k \text { with } \\
& \frac{\partial \widetilde{c}}{\partial m}=k-\frac{\partial \widetilde{h}}{\partial m}=k-\frac{1}{M_{h}} \text { and } \frac{\partial \widetilde{c}}{\partial k}=r+m-f-\mu .
\end{aligned}
$$

By Eqs. (32) and (44) the value function can be defined as follows:

$$
\Phi=\left.\max _{s, f, h} \Lambda\right|_{(42)}=\frac{U(\widehat{c}, f, k)}{\rho+m} .
$$

Noting Eqs. (28) and (44) the partial derivatives of the function Eq. (45) are

$$
\begin{aligned}
& \frac{\partial \Phi}{\partial k}=\frac{U_{c}}{\rho+m} \frac{\partial \widehat{c}}{\partial k}=\frac{U_{c}(r-\mu)}{\rho+m}=\frac{\partial \Psi}{\partial k}, \\
& \frac{\partial \Phi}{\partial m}=\frac{1}{\rho+m}\left(U_{c} \frac{\partial \widehat{c}}{\partial m}+U_{f}\right)-\frac{U}{(\rho+m)^{2}}=\frac{1}{\rho+m}\left[U_{f}+U_{c}\left(k-\frac{1}{M_{h}}\right)\right]-\frac{U}{(\rho+m)^{2}}=\frac{\partial \Psi}{\partial m} .
\end{aligned}
$$

Acknowledgements The authors are grateful to the three anonymous reviewers for constructive comments. Tapio Palokangas thanks IIASA (Laxenburg, Austria) for hospitality during his visit in July 2019 when a major part of this paper was written.

Funding Open access funding provided by University of Helsinki including Helsinki University Central Hospital.

Open Access This article is licensed under a Creative Commons Attribution 4.0 International License, which permits use, sharing, adaptation, distribution and reproduction in any medium or format, as long as you give appropriate credit to the original author(s) and the source, provide a link to the Creative Commons licence, and indicate if changes were made. The images or other third party material in this article are included in the article's Creative Commons licence, unless indicated otherwise in a credit line to the material. If material is not included in the article's Creative Commons licence and your intended use is not permitted by statutory regulation or exceeds the permitted use, you will need to obtain permission directly from the copyright holder. To view a copy of this licence, visit http://creativecommons.org/ licenses/by/4.0/.

\section{References}

Baudin T (2011) Family Policies: What does the Standard Endogenous Fertility Model Tells Us? J Public Econ Theory 13:555-593

Becker GS (1981) A Treatise on the Family. Cambridge (Mass.): Harvard University Press

Brunekreef B, Holgate ST (2002) Air Pollution and Health. Lancet 360:1233-1242

Chakraborty S (2004) Endogenous Lifetime and Economic Growth. J Econ Theory 116:119-137

Dalgaard C-J, Strulik H (2014) Optimal Aging and Death: Understanding the Preston Curve. Journal of European Economic Association (JEEA) 12:672-701

De la Croix D, Grosseries A (2012) The Natalist Bias of Pollution Control. J Environ Econ Manag 63:271-287

Ehrlich I, Chuma H (1990) A Model of the Demand for Longevity and the Value of Life Extension. J Polit Econ 98:761-782 
Galor O, Weil DN (1996) The Gender Gap, Fertility and Growth. Am Econ Rev 86:374-387

Gomez MA (2001) Subsidy Policies in a Fertility Choice Model. Atl Econ J 29:438-449

Grossman M (1972) On the Concept of Health Capital and the Demand for Health. J Polit Econ $80: 223-255$

Harford JD (1997) Stock Pollution, Child-Bearing Externalities, and the Social Discount Rate. J Environ Econ Manag 33:94-105

Harford JD (1998) The Ultimate Externality. Am Econ Rev 88:260-265

Jöst F, Quaas MF (2010) Environmental and Population Externalities. Environ Dev Econ 15:1-19

Lehmijoki U, Rovenskaya E (2010) Environmental Mortality and Long-Run Growth. In: Crespo Cuaresma J, Palokangas T, Tarasyev A (eds) Dynamic Systems, Economic Growth, and the Environment. Springer, Heidelberg, pp 239-258

Pope CA, Burnett RT, Thun MJ, Calle EE, Krewski D, Ito K, Thurston GD (2002) Lung Cancer, Cardiopulmonary Mortality, and Long-Term Exposure to Fina Particulate Air Pollution. J Am Med Assoc 287:1132-1141

Razin A, Ben-Zion U (1975) An Intergenerational Model of Population Growth. Am Econ Rev 65:923-933

Samet JM, Dominici F, Curriero FC, Coursac I, Zeger SL (2000) Fine Particulate Air Pollution and Mortality in 20 US Cities, 1987-1994. N Engl J Med 343:1742-1749

Stelter R (2016) Over-aging - Are Present-Day Human Populations Too Old? Math Soc Sci 82:116-143

Uzawa H (1968) Time Preference, the Consumption Function, and Optimum Asset Holdings. In: Wolfe J (ed) Value, Capital, and Growth, Chapter 21. Routledge, New York

Publisher's Note Springer Nature remains neutral with regard to jurisdictional claims in published maps and institutional affiliations. 